\title{
An unusual case of adrenal cortical carcinoma
}

\author{
G.R. Mufti and J. Farrell \\ Department of Urology, Glasgow Royal Infirmary, Glasgow G31 2ER, UK.
}

\begin{abstract}
Summary: We describe a case of non-functioning adrenal cortical carcinoma, which recurred 16 years after surgical adrenalectomy. In addition, the patient was successfully treated for two anatomically separate and histologically different, primary malignant tumours. This case highlights the importance of lifelong follow-up after apparently curative surgical excision of an adrenal tumour.
\end{abstract}

\section{Introduction}

Adrenal cortical carcinoma is a rare tumour accounting for only $0.02 \%$ of all malignancies. ${ }^{1,2}$ Due to the anatomical location of the adrenals the diagnosis is often delayed and a large proportion of patients present with inoperable or metastatic disease. $^{2,3}$ Therefore, it is not surprising that the overall 5 year survival rates are generally discouraging and range between 10 and $30 \%$. ${ }^{1,3}$ In those patients who present early, undergo radical surgical resection, and remain disease free for more than 2 years, the long term outlook is good. ${ }^{2,3} \mathrm{We}$ report such a case who developed a local recurrence 16 years after surgical adrenalectomy, the longest such interval from published accounts. In addition, the patient was successfully treated for two other primary malignant tumours.

\section{Case report}

A 69 year old female presented in 1989 with upper left abdominal pain. In 1962 she had undergone abdominal hysterectomy for carcinoma of the cervix. In 1973, she presented with left sided abdominal pain. Intravenous urography demonstrated a normal right kidney with a space occupying lesion involving the upper pole of the left kidney. Renal angiography confirmed it to be a left adrenal tumour. There was no clinical or biochemical evidence of a functioning adrenal medullary or cortical tumour. Exploration revealed a solid tumour of the left adrenal gland and left adrenalectomy with preservation of the left kidney was carried out. Histological examination showed it to be an adrenal cortical carcinoma. She was followed up at yearly intervals up to 1983 when

Correspondence: G.R. Mufti, M.S., M.Ch., F.R.C.S. (Ed.), Walsgrave Hospital, Coventry CV2 2DX, UK. Accepted: 19 January 1990 there was no evidence of local or metastatic disease. In 1978 the patient underwent wide excision of a malignant melanoma on the left ankle. Subsequently, block dissection of left inguinal lymph glands was carried out.

On this admission, examination was unremarkable. There was no evidence of recurrence of carcinoma cervix or malignant melanoma. Intravenous urography and ultrasonography showed a space occupying lesion involving the upper pole of the left kidney and extending to the splenic and left renal hila. The right kidney was normal. Computed tomographic (CT) scan of the abdomen confirmed the presence of a solid mass related to the upper pole of the left kidney encroaching on the renal and splenic hila and closely applied to the lower border of tail of pancreas (Figure 1). Ultrasound guided biopsy was unhelpful. Corticosteroid profile was normal. Radical en-bloc excision of the tumour, left kidney, spleen along with fat and fascia was carried out. The mass was close but not adherent to the pancreas. Histological examination confirmed it to be recurrent adrenal cortical carcinoma similar to the one removed in 1973. Follow up at 6 months after surgery did not reveal any evidence of local or metastatic recurrence.

\section{Discussion}

Two reasons prompted us to report this case. Firstly, our patient developed a recurrence 16 years after adrenalectomy. A survey of previous publications has revealed this to be the longest disease free interval reported, disease free interval being defined as the time interval between curative surgical resection and the appearance of first recurrence.

Secondly, our patient was also successfully treated for two other histologically different primary malignant tumours, one before and one after the treatment of the adrenal tumour. A higher 


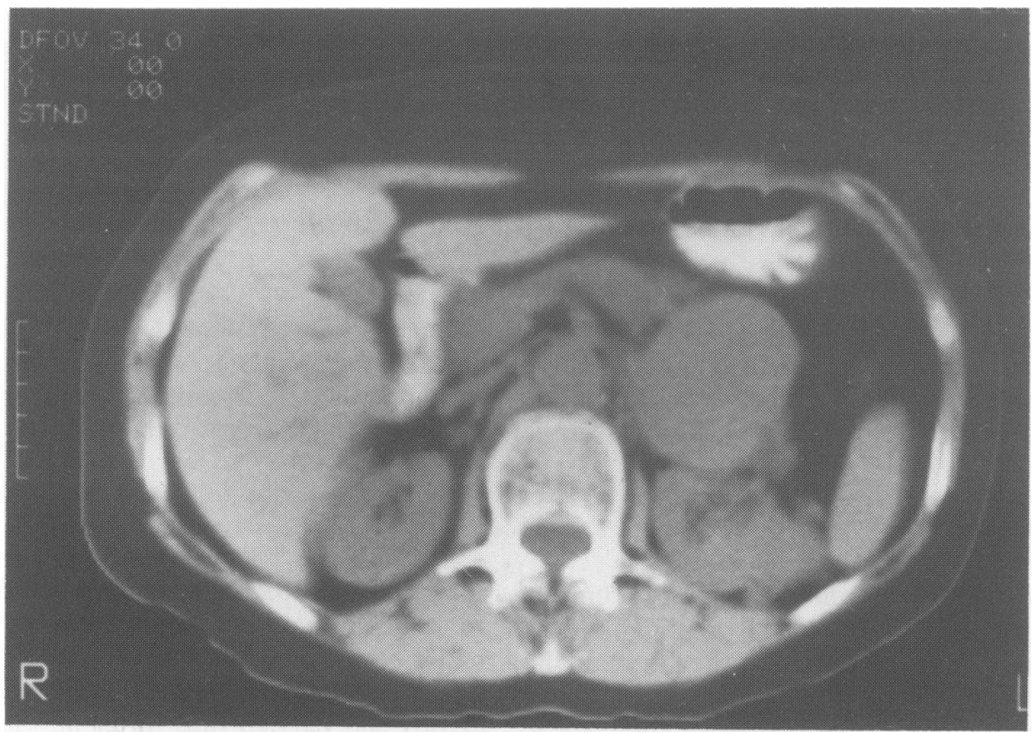

Figure 1 CT scan showing a mass in relation to the upper pole of left kidney.

incidence of other primary cancers in patients with adrenal carcinoma is recognized. ${ }^{3}$ Didolkar et al. ${ }^{3}$ reported an incidence of $24 \%$ in these patients compared to the generally accepted figure of $11 \%$ in other cancers.

Evidence suggests, that radical surgery is the only mode of treatment which offers the best chance of cure in these patients. ${ }^{1,3}$ This involves en-bloc removal of the tumour along with the kidney and adjacent involved organs. Furthermore, patients with locally recurrent disease and a long disease-free interval should be considered for surgical resection. ${ }^{4}$ It is difficult to speculate wheth er or not this patient would have developed a local recurrence if the primary surgical treatment had been more radical and included a nephrectomy. However, it is obvious from this case that a patient with adrenal cortical carcinoma who undergoes 'curative surgical resection' ought to be closely followed up for life, not only for signs of recurrence but also to detect other primary cancers at an early stage.

\section{References}

1. Samaan, N.A. \& Hickey, R.C. Adrenal cortical carcinoma. Semin Oncol 1987, 14: 292-296.

2. King, D.R. \& Lack, E.E. Adrenal cortical carcinoma. A clinical and pathological study of 49 cases. Cancer 1979, 44: 239-244.

3. Didolkar, M.S., Bescher, R.A., Elias, E.G. \& Moore, R.H. Natural history of adrenal cortical carcinoma. A clinicopathological study of 42 patients. Cancer 1981, 47: 2153-2161.

4. Bradley, E.L. Primary and adjunctive therapy in carcinoma of adrenal cortex. Surg Gynecol Obstet 1975, 141: 507-511. 